\title{
A Simplified Phase Model for Simulation of Oscillator-Based Computing Systems
}

\author{
YAN FANG, VICTOR V. YASHIN, BRANDON B. JENNINGS, DONALD M. CHIARULLI, \\ and STEVEN P. LEVITAN, University of Pittsburgh
}

\begin{abstract}
Building oscillator-based computing systems with emerging nano-device technologies has become a promising solution for unconventional computing tasks like computer vision and pattern recognition. However, simulation and analysis of these computing systems is both time and compute intensive due to the nonlinearity of new devices and the complex behavior of coupled oscillators. In order to speed up the simulation of coupled oscillator systems, we propose a simplified phase model to perform phase and frequency synchronization prediction based on a synthesis of earlier models. Our model can predict the frequency-locking behavior with several orders of magnitude speedup compared to direct evaluation, enabling the effective and efficient simulation of the large numbers of oscillators required for practical computing systems. We demonstrate the oscillator-based computing paradigm with three applications, pattern matching, convolution, and image segmentation. The simulation with these models are respectively sped up by factors of 780,300 , and 1120 in our tests.
\end{abstract}

CCS Concepts: $\bullet$ Hardware $\rightarrow$ Emerging technologies; $\bullet$ Computing methodologies $\rightarrow$ Computer vision

Additional Key Words and Phrases: Non-Boolean computing, oscillator-based computing, coupled oscillators, phase model, synchronization

ACM Reference Format:

Yan Fang, Victor V. Yashin, Brandon B. Jennings, Donald M. Chiarulli, and Steven P. Levitan. 2016. A simplified phase model for simulation of oscillator-based computing systems. J. Emerg. Technol. Comput. Syst. 13, 2, Article 14 (November 2016), 20 pages.

DOI: http://dx.doi.org/10.1145/2976743

\section{INTRODUCTION}

Pursuing high-density, low-power, high-speed computing systems for the post-CMOS era drives researchers to exploit the potential of emerging nano-device technologies. Nonetheless, with the constraint of building computing systems with traditional Boolean logic, it is difficult for these new technologies to outperform prevailing CMOS technology in computing speed or power dissipation [Hutchby et al. 2008; Bourianoff et al. 2008]. Thus, investigations of nanotechnology to replace the CMOS transistor for Boolean computing structures with a more energy-efficient device have been largely unsuccessful. However, because some of these devices possess characteristics of multistate response that differ from traditional CMOS transistors, it is possible to utilize

This work was supported in part by the National Science Foundation under grants DMR-1344178 and grant CCF-1317373.

Authors' addresses: Y. Fang, B. B. Jennings, and S. P. Levitan, Department of Electrical and Computer Engineering, University of Pittsburgh, 1238 Benedum Hall, Pittsburgh, PA 15261; emails: \{yaf13, bbj5, levitan\}@pitt.edu; V. V. Yashin, Department of Chemical \& Petroleum Engineering, University of Pittsburgh, 940 Benedum Hall, Pittsburgh, PA 15261; email: vvy1@pitt.edu; D. M. Chiarulli, Department of Computer Science, University of Pittsburgh, 6137 Sennott Square Pittsburgh, PA 15260; email: don@cs.pitt.edu.

Permission to make digital or hard copies of part or all of this work for personal or classroom use is granted without fee provided that copies are not made or distributed for profit or commercial advantage and that copies bear this notice and the full citation on the first page. Copyrights for third-party components of this work must be honored. For all other uses, contact the Owner/Author.

Copyright 2016 held by Owner/Author.

1550-4832/2016/11-ART14

DOI: http://dx.doi.org/10.1145/2976743 
them effectively in non-Boolean information processing systems for applications like neural computing, computer vision, and pattern recognition.

Based on recent advances in emerging nano-devices such as spin torque oscillators (STO) [Pufall et al. 2005; Locatelli et al. 2014], resonate body transistor oscillators (RBO) [Weinstein and Bhave 2010], and vanadium oxide oscillators $\left(\mathrm{VO}_{2}\right)$ [Shukla et al. 2014], systems built from coupled nano-oscillators have become promising candidates for next generation computing structures used in intelligent information processing [Cotter et al. 2014]. Inspired by the interaction between neural oscillation that occurs at different time scales in biological systems, Hoppensteadt and Izhikevich [2000] developed an associative memory model of coupled oscillators using phase-locked loops and provided examples of how this dynamic system performs recognition by forming attractor basins at the minima of a Lyapunov energy function. Locally coupled oscillator arrays inspired by visual cortex can also perform image segmentation and scene grouping [Wang and Termani 1995].

Besides biologically inspired systems, pattern matching, or nearest neighbor searching is another effective and useful application of oscillator-based computing. Similar to an associative or content addressable memory, pattern matching is performed between an input vector and a pool of stored vectors, where one or several closest vectors are required to be retrieved. The distance metric for vector comparison in pattern matching can be obtained by observing how well oscillators synchronize with each other.

The essential idea of utilizing coupled oscillator systems to perform computation lays in the energy transfer in a dynamical system. Initialized with input information, a number of oscillators interact and exchange energy with each other, making the whole dynamical system converge from a perturbed state to a stable state. This process brings several advantages to oscillator based pattern matching. First, it provides a higher level multi-dimension norm like the Euclidean distance between sets of input vectors, compared to the Hamming distance computed with exclusive-OR Boolean operations. Second, it is suitable for high-dimension large data sets due to its scalability and the fact that the degree of match spans all of the dimensions of an input vector without any arithmetic calculations [Levitan et al. 2012]. Third, with the high frequency of new devices like STOs, the systems can converge quickly. Although systems built from these nano-devices have not been realized to date, prototypes using traditional circuits have been built and simulated [Shibata et al. 2012]. Further, simulations of systems using nano-oscillators have shown promising results [Csaba and Porod 2013].

In general, the configuration of oscillator-based computing systems, such as network topology, input pattern representation, detection of output results and "degree of match", has numerous varieties; and, the design of these circuits can be tailored by specifications from system designers. Recently, research has been conducted not only from the perspective of devices and circuits [Kabir and Stan 2014; Shibata et al. 2012], but also at the level of algorithms and architectures [Levitan et al. 2012; Csaba and Porod 2013].

In order to design and build systems based on coupled oscillator networks, we need a good understanding of coupled oscillator systems, including the synchronization and de-synchronization between oscillators, the prediction of the coupled oscillators' frequencies, and their relation to the degree of match function in pattern matching. Unfortunately, accurate simulation and analysis of such a computing system from the device level to the architecture level is difficult for current EDA/CAD tools for two reasons. One is the complexity of the nano-oscillators' device model and the other is that the interactions due to coupling makes the dynamics difficult to predict. Thus, circuit and system designers cannot model systems at very large scales, while algorithm designers and architects can only use approximations of the oscillators' real behaviors. 
To address this problem, we need to focus on modeling the behavior of coupled oscillators. While coupled oscillators have been studied since 1665 by Christiaan Huygens, in the last few decades, Winfree [1967] and Kuramoto [1984] made fundamental contributions to the understanding of these systems. Hoppensteadt and Izhikevich [1997] studied weakly coupled oscillator networks in their book from the perspective of neuroscience and dynamic systems, and they also unified previous work by using the idea of the phase resetting curve (PRC) [Izhikevich 2007]. Recently, Demir et al. [2000] proposed a nonlinear phase model based on a perturbation projection vector (PPV) to study the perturbations due to noise of electronic oscillators [Demir et al. 2000] and used this to model coupled oscillators. These models were widely used in modeling the injection locking of oscillators to external signals [Lai and Roychowdhury 2005]. Mafizzoni developed this model one step further and analyzed weakly coupled oscillators from the perspective of multi-frequency analysis [Maffezzoni 2010].

The rest of this article is organized as follows. First, we illustrate the concept of oscillator-based computing by giving examples of three oscillator computing applications. Second, we study coupled oscillators using different phase models and demonstrate the equivalency between them. Then, we combine ideas from these models to abstract a simplified model for oscillator-based pattern-matching operations. Finally, we provide comparative simulation results and conclude with observations about the effectiveness of our model and future work.

\section{OSCILLATOR COMPUTING PARADIGMS}

In this section, we give an introduction to three oscillator computing paradigms for different applications in pattern matching and image processing. In an image-processing pipeline for object recognition, image segmentation belongs to the preprocessing stage of the front-end; pattern matching can serve as the classifier in the back-end; and the convolution operation is widely used in object recognition models like convolutional neural networks and the HMAX model [Krizhevsky et al. 2012; Serre and Riesenhuber 2004].

\subsection{Pattern Matching}

Pattern matching is basically a nearest neighbor search operation and the patterns can be images, features, or vectors, depending on different applications. Figure 1(a) shows an abstract view of a "content addressable" associative memory [Foster 1976]. Here, a set of associative storage words constitute a single large memory. The set of pattern vectors to be matched (e.g., handwritten digits) are first stored in the memory as template pattern vectors. In this article, we define "templates" as the stored pattern vectors of associative memory. Depending on the application, codes that correspond to the templates are also stored. Matching operations proceed by broadcasting (on a bus) input vectors to all words in the memory. Then, each word performs a local comparison or match operation. This local match operation is relative, generating a "degree of match" between the input pattern vector and the local template stored in the word. Next, the degree of match from each word is compared by a global resolution function, and the best matching result is output.

Figure 1(b) shows the architecture of a single Associative Memory module composed of coupled oscillators. The module contains multiple clusters of oscillators connected to each other with a "star" structure around a summing node. Each cluster has a template memory, $T^{j}$, that holds a vector of $t_{i}^{j}$ values ( $j$ is the index of the template vectors). The template memory can be either digital or analog. For digital memories, we would need D/A converters to provide the template values to the oscillator clusters for comparison. Once the templates are loaded, comparisons happen between every oscillator cluster 


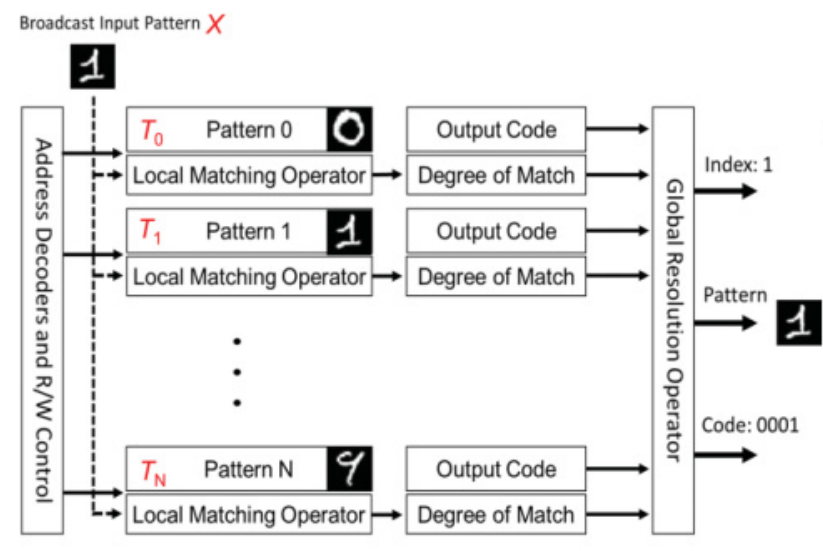

(a)

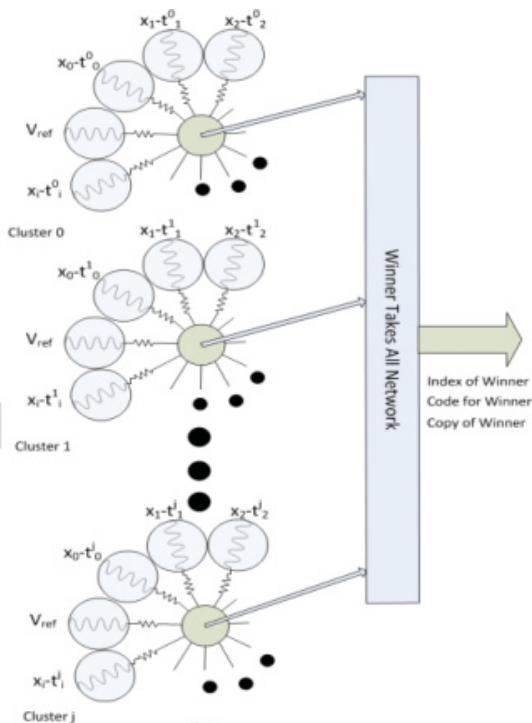

(b)

Fig. 1. (a) Associative memory model for handwritten digits; (b) Coupled oscillator-based associative memory module.

and the input pattern vector, $X$. This process starts from initializing the frequencies or phases of each oscillator with the difference between element values of $X$ and $T^{j}$. The degrees of match (DoM) between the input pattern and the stored templates are then determined by how well a cluster of oscillators synchronizes. If $X$ and $T^{j}$ are similar to each other, the oscillators' final frequencies or phases are the same or close to each other. Otherwise, the oscillator cluster will generate a more chaotic desynchronized signal. In other words, the coupled oscillator system behaves like a kernel that gives the distance measure between two vectors, which is very similar to squared Euclidean distance. A degree of match detector circuit is required to measure the DoM from the summing node of each oscillator cluster and feeds its output to a winner-takes-all network to choose the best match among all clusters composing the memory. As the system scales, the performance and power consumption depends on the characteristics of oscillator devices.

This oscillator-based associative memory system is compatible with different types of oscillators. For instance, recently a low-power design for this computing paradigm was implemented with Spin Hall Effect STO CMOS and interface circuits [Fan et al. 2015]. Here we use ring oscillators because they can be efficiently implemented with CMOS techniques and their behavior was analyzed in previous work [Lai and Roychowdhury 2005]. Figures 2(a) and 2(b) gives an example of the circuit design of a coupled ring oscillator cluster for pattern matching. For these oscillators, the frequency of each oscillator is adjusted by two input control voltages and the coupling node is the output node of the third inverter. The frequency control voltages, $V$, and $\bar{V}$ are the pairwise difference of vectors, $\left|X-T^{j}\right|$. The oscillators are coupled to each other through a common node, where the coupling components could be resistor or capacitor, where larger resistors would give weaker coupling strength. The output signal of the common node is amplified and rectified to form a DoM detection circuit. Other DoM detection techniques and circuits can be found in Shibata et al. [2012]. In general, the design of the DoM detection circuit is a function of the frequency and wave-shape of the oscillators and the coupling circuit. Figure 2(c) shows how close the distance metric 


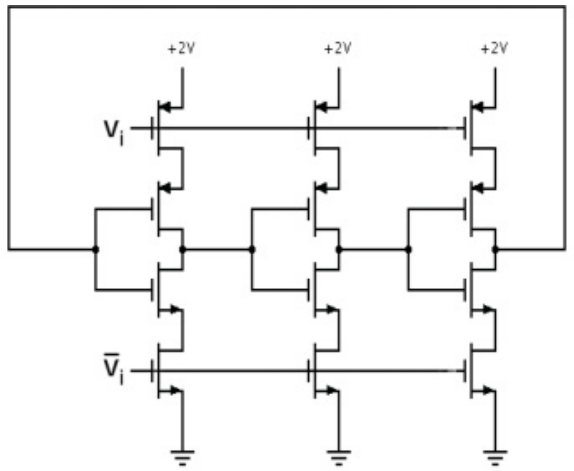

(a)

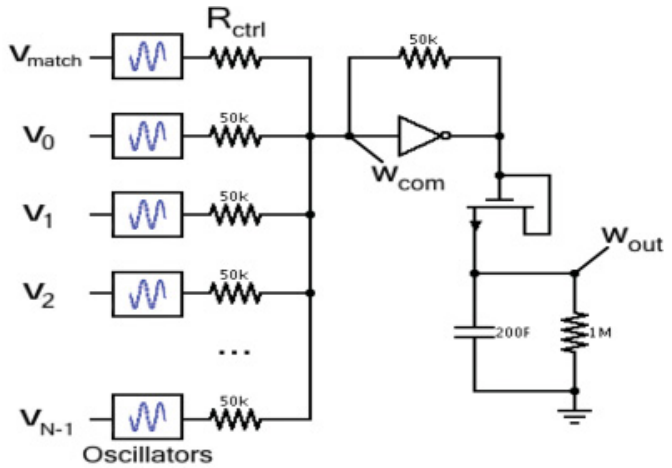

(b)

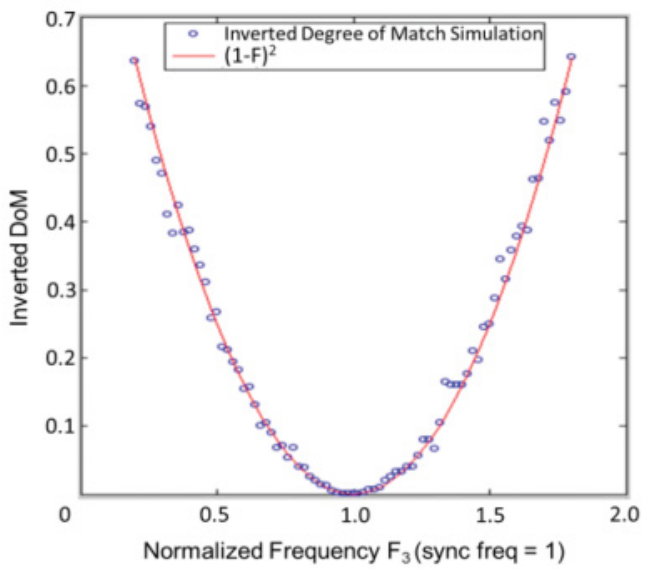

(c)

Fig. 2. Coupled oscillator system. (a) Voltage controlled ring oscillator; (b) Oscillators coupled together through one common node with DoM detection circuit; (c) Simulation of a three-oscillator cluster by sweeping one frequency, the scatter plot of 1-DoM is against the curve fit of a quadratic model.

is to the squared Euclidean distance for an optimized design. In this three-oscillator system, one oscillator's frequency, $\mathrm{F}_{3}$, is swept while the other two remain fixed. The scatter plot of $(1-D o M)$ is generated by the DoM detection circuit in Figure 2(b). It is compared against the quadratic curve fit model $\left(1-\mathrm{F}_{3}\right)^{2}$. In this diagram, frequencies are normalized to 1 .

\subsection{Convolution}

The second application example is the use of oscillators to perform the convolution of vectors. As we discussed above, the coupled oscillator clusters can provide a squared Euclidean distance metric. This enables a large variety of image processing algorithms and vector distance based classifiers to be accelerated or in some cases directly computed using oscillator DoM primitives.

However, it is also possible to directly implement a vector convolution primitive with oscillators by using three oscillator clusters. We observe that the oscillator DoM circuits give us an inverted normalized squared Euclidean distance $\left(\mathrm{L}_{2}{ }^{2}\right)$. By expanding and rearranging Equation (1), we can derive an expression for the convolution of vectors $\mathrm{A}$ 


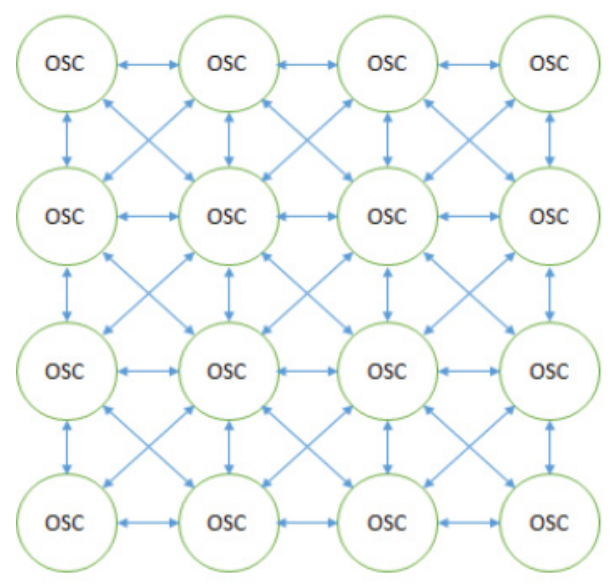

(a)

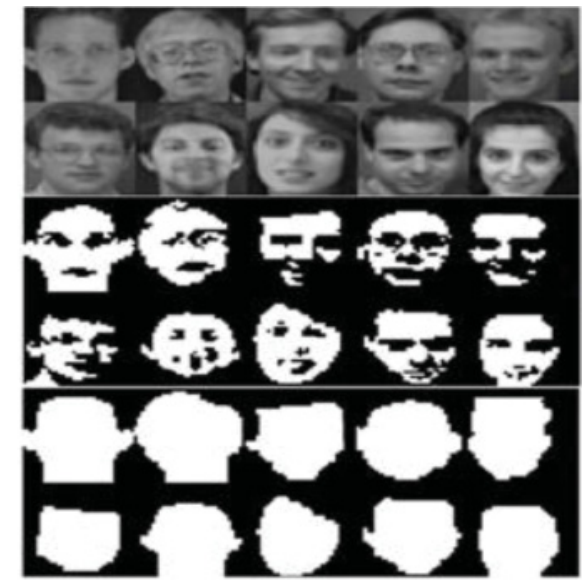

(b)

Fig. 3. (a) Nearest Neighbor Network with bi-directional coupling; (b) Segmentation on face image dataset. Top: face images; Middle: fine grain segmentation; Bottom: coarse grain segmentation.

and $\mathrm{B}$ in terms of $\mathrm{L}_{2}^{2}$,

$$
\begin{aligned}
& L_{2}^{2}(A, B)=(A-B)^{2}=A^{2}-2 A B-B^{2} \\
& \frac{L_{2}^{2}(A, B)-L_{2}^{2}(A, 0)-L_{2}^{2}(B, 0)}{-2}=A B
\end{aligned}
$$

In this implementation, the first cluster computes the $\mathrm{L}_{2}{ }^{2}$ distance of $\mathrm{A}$ and $\mathrm{B}$, the second cluster computes the $\mathrm{L}_{2}{ }^{2}$ of $\mathrm{A}$ and 0 , and the third of $\mathrm{B}$ and 0 . Subtraction, division by two, and inversion are implemented in analog support circuitry. An oscillator implementation of a convolution kernel enables a wide variety of signal processing and image processing primitives to be implemented or accelerated. This includes key kernels for filtering, spectral transforms, and convolutional neural networks [Chiarulli et al. 2015].

\subsection{Image Segmentation}

The third example of an oscillator computing application is designed particularly for image segmentation [Fang et al. 2014]. The structure of this oscillator network is a two-dimensional array of coupled oscillators where each oscillator is coupled with its eight neighbors, shown in Figure 3(a). For the image segmentation task, the oscillator network is configured such that it has the same size as the input image and each oscillator corresponds to one pixel. We initialize the oscillation of each oscillator with a frequency, depending on the intensity information of each pixel.

After initialization of the oscillator network, the oscillators try to synchronize with their neighbors and their frequencies begin to shift towards each other. If the pixels belong to the same region, their intensity values are typically close to each other. Thus, the corresponding oscillators will synchronize with each other and lock to the same frequency. Otherwise, their frequencies are too far apart to synchronize and they maintain their own frequency. As a result, the oscillators in the network are clustered into groups. Oscillators within each group share the same or similar frequency. In this way, pixels are clustered into different regions.

The coupled oscillators perform pixel clustering based on two criteria: spatial locality and intensity similarity of the pixels. For our oscillator network architecture, the 
coupling strength between oscillators can be used to tune the networks to reduce noise sensitivity while maintaining good segment borders. However, a "good" segmentation of the image is specified by the requirements of later image processing tasks, reinforcing a need for an abstract definition for comparison purposes. In Figure 3(b), we illustrate two segmentation tasks. In the first, with lower coupling strength, we generate fine grain segments from the images highlighting facial detail. In the second, using stronger coupling, we extract course grain facial shapes from the input images. In this paradigm, oscillators perform pixel-level clustering automatically with high efficiency. The system can be adjusted flexibly to satisfy different segmentation resolution for various purposes of segmentation.

\section{PHASE MODELS}

As we discussed in the introduction, the simulation and analysis of these oscillator computing paradigms are usually difficult and time-consuming. In this Section, we try to address the problem by studying the phase models of oscillators, which can be used to effectively describe this behavior. We first review phase models proposed in previous work, including the PPV model [Demir et al. 2000], Winfree's and Malkin's approaches summarized by Izhikevich [2007]. By discussing the relation between these models and combining their advantages, we develop our new model.

\subsection{PPV Model}

We start with the Phase Projection Vector (PPV) model, since it is derived from the demand for a theoretical analysis of oscillator circuits perturbed by noise and therefore is easier to understand from an electrical engineering perspective compared to the other models [Demir et al. 2000].

The PPV model starts with the general differential equation of an oscillator,

$$
\dot{x}(t)=f(x(t))+P(t)
$$

where $x(t)$ is the vector of oscillation states and $\dot{x}(t)$ is their derivatives. In real circuits, these states are usually the voltages or currents of nodes. $P(t)$ represents an external perturbation on the oscillation, which can be noise, signal injection, or in this case, a coupling term from other oscillators. $f$ is the nonlinear function that describes the oscillation and $t$ is time. We assume the perturbation is small and it only changes the phase of the oscillators. Then, the response solution from this equation can be written as,

$$
x_{c}(t)=x_{s}(t+\alpha(t))
$$

where $x_{s}(t)$ is the oscillator's natural response without any perturbation, namely $P(t)=$ 0 , while $x_{c}(t)$ is the response with perturbation. $\alpha(t)$ represents the time shift of phase that is caused by the perturbation. Hence Equation (2) reveals the phase relation between the natural response and the perturbed response of the oscillator. According to the PPV model, $\alpha(t)$ can be obtained by solving the equation,

$$
\dot{\alpha}(t)=\Gamma(t+\alpha(t)) P(t)
$$

where $\Gamma(t)$ is the perturbation projection vector (PPV) that describes the perturbation response of the oscillators. The PPV can be thought of as the time-varying sensitivity of the induced time shift to the given injected perturbation [Lai and Roychowdhury 2005]. The theoretical derivation and proof of the PPV method can be found in Demir et al. [2000]. The PPV, $\Gamma(t)$, is usually a periodic function that can be obtained either numerically or analytically. After acquiring $\Gamma(t)$, we know the time shift of phase $\alpha(t)$ from the solution of Equation (4) and thus can solve Equation (3). This model is referred as analytical PPV in this article. 
This model actually does not predict the "phase change" but a time shift function of oscillation response. Thus, it is difficult for us to use this model to directly predict the frequency shift in a coupled oscillator system. The next models we introduce provide us further insight.

\subsection{Izhikevich's Model}

In Izhikevich [2007], the phase model of weakly coupled nonlinear oscillators is explored from an abstract view, by unifying some earlier models. Differing from the PPV model, this model assumes all the oscillators share the same free-running frequency, so the "phase" of nonlinear coupled oscillators can be normalized and defined as,

$$
\theta(t)=t+\varphi(t)
$$

Taking the derivative of both sides, we have,

$$
\dot{\theta}(t)=1+\dot{\varphi}(t)
$$

In Equations (5) and (6), $\theta(t)$ is the defined phase, a periodic function with period $\mathrm{T}=1 . \varphi(t)$ is called the phase deviation, caused by the coupling from other oscillators. We notice that when there is no coupling term, the phase term is simply time, $t$, and the free-running frequency is normalized to 1 . The derivative $\dot{\varphi}(t)$ represents the change of phase deviation, namely the frequency shift, due to the coupling effect.

In order to map this model to various nonlinear oscillators, the key point lies in the phase deviation $\varphi(t)$, described by:

$$
\dot{\varphi}(t)=Q(\theta(t)) P(\mathrm{t})
$$

which has a similar form to Equation (4). $P(t)$ is the same external injection signal to the oscillators (i.e., the coupling term). The function $Q(\theta)$ is called the phase response curve or phase resetting curve (PRC), representing how sensitive the phase deviation is in response to $P(t)$ at a specific phase $\theta(t)$. Thus (6) can also be written as,

$$
\dot{\theta}(t)=1+Q(\theta(t)) P(t)
$$

The mechanisms of phase and frequency interactions of coupled oscillators revealed by this equation was discovered multiple times in the early studies of oscillator phase models and some researchers named it PRC in different ways and exploited different methods to derive and utilize it.

A theorem first proposed by Malkin in 1949 and later abstracted by Hoppensteadt and Izhikevich [1997] indicates that $Q(\theta)$ can be computed by solving the linear adjoint equation,

$$
\dot{Q}(t)=-\mathcal{J}[f(x(t))]^{T} Q
$$

where $\mathcal{J}[f(x(t))]^{T}$ is the transposed Jacobian matrix of the oscillation function $f$ in (1). This theorem is identical to Kuramoto's approach in 1984 where the gradient of phase plays the role of PRC.

In Winfree's work (1967), PRC was experimentally approached by applying a pulse stimuli with amplitude A. Then a function called the linear response or sensitivity function $Z(\theta)$ was measured by observing the phase shift caused by the stimuli, and $\mathrm{PRC}$ is $Z(\theta)$ divided by amplitude $A$.

$$
\operatorname{PRC}(\theta) \approx \frac{Z(\theta)}{A}
$$

This method is flexible and intuitive. However, being experimental, it suffers from more variation and inaccuracy. Nonetheless, it is useful when the nonlinear oscillator equations is not differentiable or continuous. 


\subsection{Our Simplified Model}

From the previous models discussed above, if we pair Equations (2) and (5), (3) and (6), and (4) and (7) and compare them, we can notice that they have the same pattern because the intrinsic method behind these models is the same, which is to quantify how the oscillation is affected by the external perturbation. However, these models use different methods to calculate this term, either an analytical derivation or a numerical measurement. In addition, the PPV model studies the oscillation variables themselves while Izhikevich's model focuses on the "phase" of nonlinear oscillators.

From the view of solving the practical problem we are addressing, the pattern matching operation is performed by frequency shifting or frequency locking of oscillators caused by coupling. The elements of the pattern vectors are represented by frequencies and the degree of match is evaluated by how well the oscillators synchronize. Thus, for the purpose of predicting the frequencies of coupled oscillators, we introduce the phase definition idea from Izhikevich's model into the PPV model.

We assume we have $n$ oscillators with different frequencies: $\omega_{0}, \omega_{1}, \omega_{2} \ldots \omega_{\mathrm{n}-1}$. Since equations (5) and (6) require that oscillators run at the natural frequencies normalized to 1 , we scale these frequencies to $1, \lambda_{1}, \lambda_{2} \ldots \lambda_{\mathrm{n}-1}$, where $\lambda_{i}=\frac{\omega_{i}}{\omega_{0}}=\frac{\mathrm{T}_{0}}{\mathrm{~T}_{i}}$. So for an arbitrary oscillator $i$, in the PPV model, Equation (3) can be changed into the phase form similar to (5) with

$$
\begin{gathered}
\theta_{i}(t)=\lambda_{i}(t+\alpha(t))=\lambda_{i} t+\varphi_{i}(t) \\
\varphi_{i}(t)=\lambda_{i}(\alpha(t))
\end{gathered}
$$

Equation (11) indicates the relation of phase deviation in Izhikevich's model and time shift of phase in the PPV model. Taking the derivative of (10), we get,

$$
\dot{\theta}_{i}(t)=\lambda_{i}+\lambda_{i} \dot{\alpha}(t)
$$

substituting with (4) we have,

$$
\dot{\theta}_{i}(t)=\lambda_{i}+\lambda_{i} \Gamma\left(\theta_{i}(t)\right) P_{i}(t)
$$

where $\Gamma\left(\theta_{i}(t)\right)$ is still the PPV in (4) but determined by the phase, instead of time. This equation transfers the PPV from a time domain to the phase domain and replaces the simulation time span into the number of oscillation cycles. $P_{i}(t)$ is the coupling term here in this model, defined as:

$$
P_{i}(t)=\sum_{j=0}^{n} g_{i j} x_{s}\left(\theta_{j}(t)\right)
$$

where $g_{i j}$ is the pair-wise coupling coefficient and $j$ is the index of other oscillators in the system. Therefore, solving (13) can provide the frequency and phase response of a coupled oscillator system. In our simplified model, the PPV function is also equivalent to the PRC function obtained from other methods. In the next section, we give examples of these methods. We note that it is useful to have several methods available because some methods might prove inaccurate or fail to converge for specific nonlinear oscillator systems.

\subsection{Oscillator Examples}

In this section, we use ideal ring oscillators as an example and demonstrate three different methods to obtain their PPV/PRC function. A simple ring oscillator consists of three inverters and RC circuits, shown in Figure 4(a) with its analytical model given in Lai and Roychowdhury [2005], presented as below. The voltage derivatives of the 

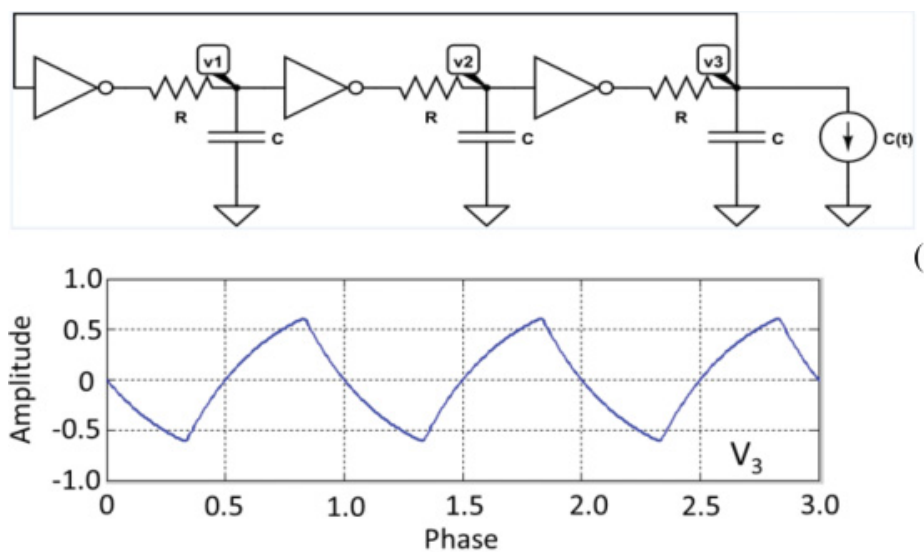

(a)

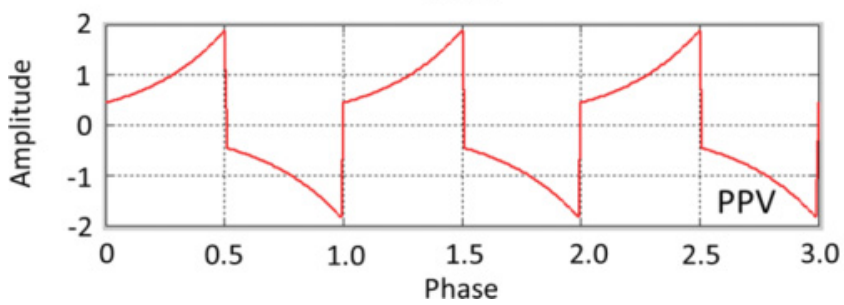

(b)

Fig. 4. Ring oscillator model. (a) Simple schematic model; (b) Output waveform and (PPV) at node v3. three nodes are:

$$
\dot{v}_{i}(t)=\frac{f\left(v_{i^{p}}(t)\right)-v_{i}(t)}{R C}, i=1,2,3
$$

where $i^{p}$ is the previous node of $i$ and $f(v(t))$ is the simplified response of an inverter:

$$
f(v)=\left\{\begin{array}{l}
+1, \text { if } v>0 \\
-1, \text { otherwise }
\end{array}\right.
$$

By normalizing the standard frequency and period to 1 , we can write the voltage state response of three nodes as,

$$
\begin{aligned}
& v_{1}(t)=\left\{\begin{array}{c}
1-\psi e^{-\gamma t}, 0 \leq t \leq 1 / 2 \\
-1+\psi e^{-\gamma\left(t-\frac{1}{2}\right)}, 1 / 2 \leq t \leq 1
\end{array}\right. \\
& v_{2}(t)=v_{1}\left(t-\frac{2}{3}\right), v_{3}(t)=v_{1}\left(t-\frac{1}{3}\right)
\end{aligned}
$$

where $\psi=\frac{1+\sqrt{5}}{2}, \gamma=6 \ln (\psi)$. Because the frequency here is $1, \mathrm{RC}=1 / \gamma$. Similarly, the PPV equation can be analytically solved as,

$$
\begin{gathered}
\Gamma_{1}(t)=\Gamma_{3}\left(t-\frac{2}{3}\right) \\
\Gamma_{2}(t)=\Gamma_{3}\left(t-\frac{1}{3}\right) \\
\Gamma_{3}(t)=\gamma^{-1} \frac{1+\psi^{3}}{4-2 \psi^{3}}\left(\psi+2\left[-u(t)+\left(-1+2 \psi^{-1}\right) u\left(t-\frac{1}{2}\right)\right]\right) e^{\gamma t}
\end{gathered}
$$




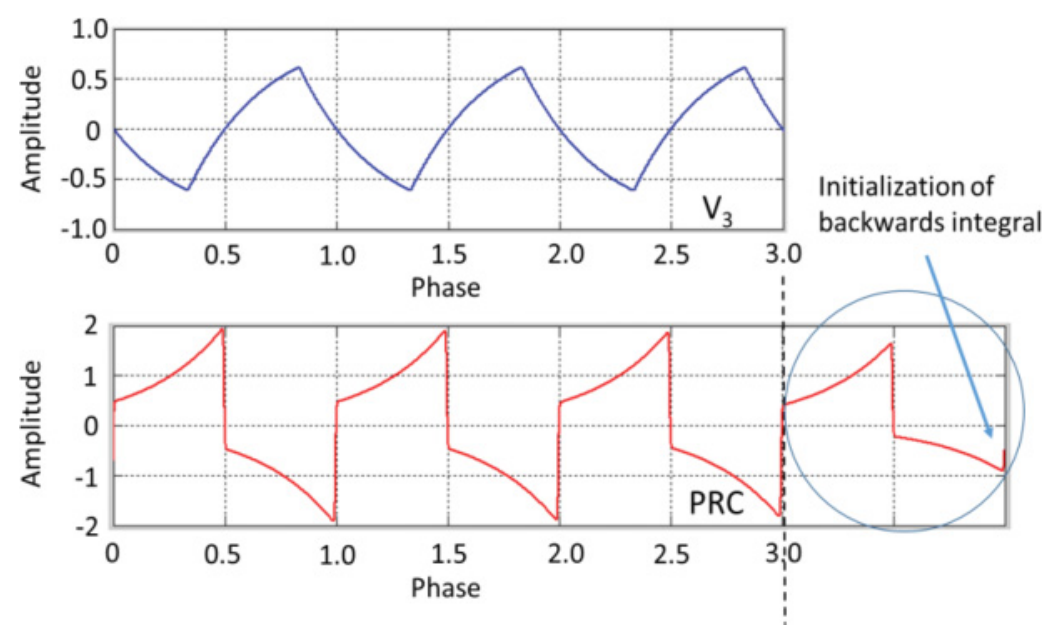

Fig. 5. Malkin's approach for PRC. (x-axis: phase, y-axis: amplitude) and output waveform at node v3 and PRC obtained from backward integral.

Figure 4(b) shows the waveform of oscillation response and PPV of node v3. From this plot, we see that the PPV function for ring oscillators is periodic and non-linear. Sometimes it is difficult to obtain the state response and PPV function directly from the ODE. For these cases, we can obtain the corresponding PRC by applying Malkin's approach numerically. Solving Equation (9) is actually very similar to the analytically derivation of PPV in Maffezzoni [2010]. However, when the analytical method does not work, we can use a technique called backward integration to obtain the Jacobian matrix [Izhikevich 2007]. Figure 5 shows the results of PRC from this method with a backwards integral of 4 cycles. The last cycle of PRC curve is inaccurate due to the special integral technique and usually should be discarded. The PRC in the first cycle is the one used.

In a few cases when a system's Jacobian matrix does not exist, Winfree's approach can be the only choice, especially for those nonlinear oscillators with complex mathematical models, even though this method is "experimental" and tends to be inaccurate. As an example, we apply Winfree's method for the ring oscillator by adding a pulse stimulus with small amplitude and measuring the phase resetting curve step by step. Figure 6 illustrates the PRC generated by Winfree's method with different stimuli amplitudes. The glitches in the curve show the problems of this approach.

However, it is worth noting that the PRC amplitude here is proportional to the stimuli amplitude by factor of 2, which not only corresponds to Equation (10) but also fits the PPV/PRC amplitude of the previous two methods in Figure 5(b) and Figure 6(b). These three examples for phase deviation of the ring oscillator indicate that PPV and PRC functions are identical to each other and enhance the foundation of our model.

\section{EXPERIMENTS AND SIMULATION}

\subsection{System Configuration}

In this section, we apply our simplified phase model to coupled oscillator systems and analyze their synchronization behavior. We also compare the performance and efficiency of the models obtained by the different approaches as well as accuracy and speedup compared to the direct simulation of the oscillator systems.

As we discussed in the previous section, a weakly coupled system, such as shown in Figure 2, can be simply described by Equations (14) and (15). In this structure, the 

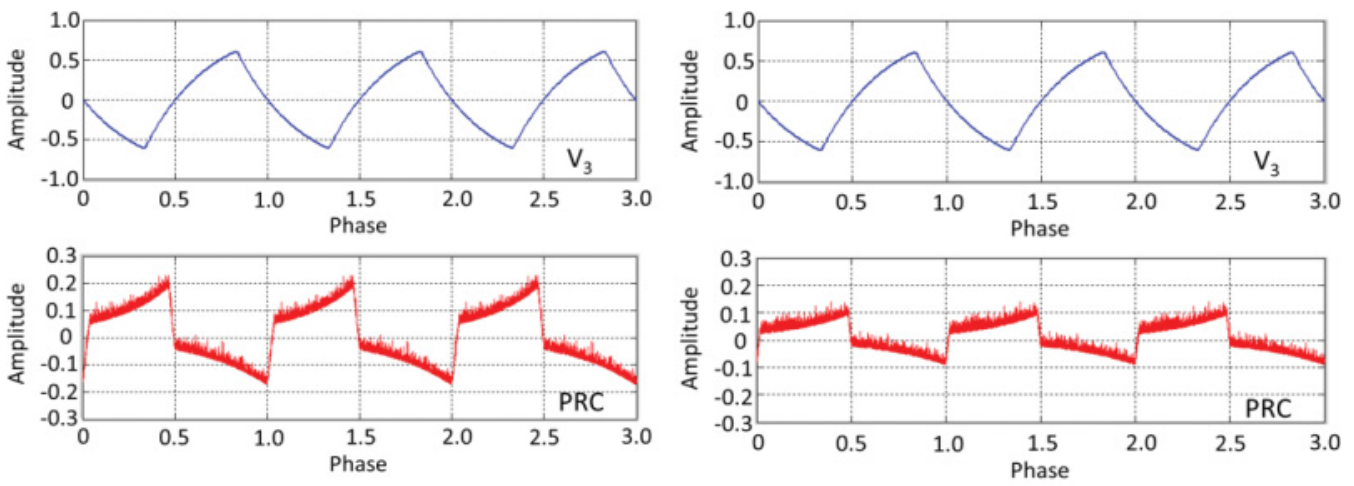

(a)

(b)

Fig. 6. Winfree's approach for PRC, (a) Stimuli Amplitude =0.1, PRC Amplitude = 0.2; (b) Stimuli Amplitude $=0.05$, PRC Amplitude $=0.1$.
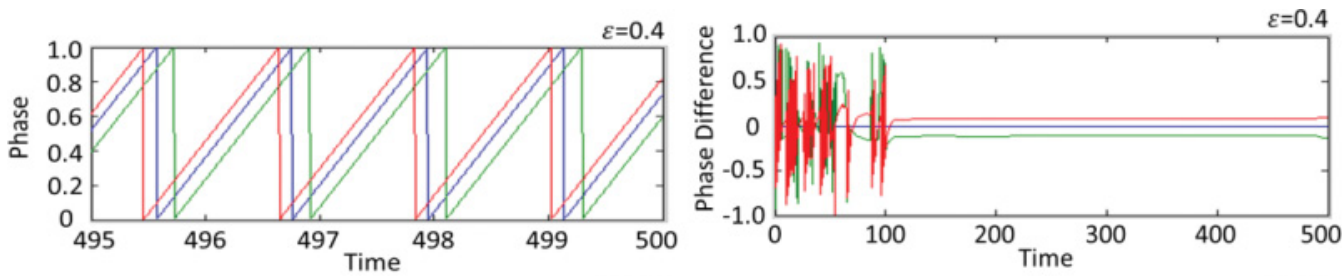

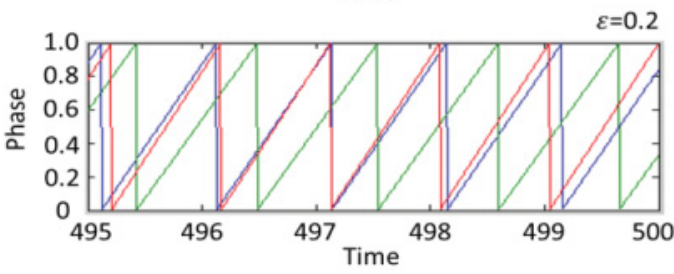

(a)

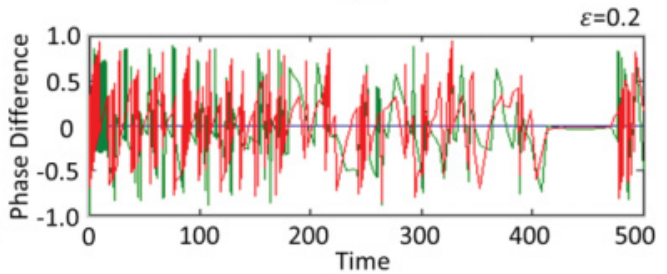

(b)

Fig. 7. Comparing simulations of three coupled oscillators with natural frequencies $\lambda=[1.0,0.95,1.05], \varepsilon=$ 0.4 and 0.2. (a) Wrapped phase waveform of final stage; (b) Evolution of phase differences between oscillators.

coupling strengths between oscillators are identical. Thus, for each oscillator at node v3 in Figure 4 we have,where $\varepsilon$ is the coupling coefficient.

$$
\dot{\theta}_{i}=\lambda_{i}+\varepsilon \lambda_{i} \Gamma_{\text {node } 3}\left(\theta_{i}\right) \sum_{j=0}^{n} v_{\text {node3 }}\left(\theta_{j}\right), i, j \in[1, n]
$$

\subsection{Oscillator Behavior Analysis}

We start with a three-oscillator system $(n=3)$. It is convenient for us to predict the final frequency of each oscillator by solving (20) numerically. If $\dot{\theta_{1}}=\dot{\theta_{2}}=\dot{\theta_{3}}$, the system is synchronized and frequency locked. We use Matlab to run a simulation of Equation (20). Figure 7 illustrates an example of locking and non-locking systems. For these examples, we set the initial frequencies to be: $\lambda=[1,0.95,1.05]$ but use two different $\varepsilon, 0.2$ and 0.4 . In the left diagram Figure 7(a), we give a zoom-in observation of wrapped phases in the final steps of simulation, where $\varepsilon=0.4$ results the locked final frequencies [0.8717 0.87170 .8717 ] while $\varepsilon=0.2$ generates desynchronized final frequencies [0.9644 0.9298 1.0263]. Figure 7(b) shows the phase differences between 
three oscillators. In the first case, the phase difference is constant while it is unstable in the second case.

From these simulation results, it is worth noting that the conditions for frequency locking are determined by the coupling coefficient $\varepsilon$ and the scaling ratio between each oscillators' free running frequency $\lambda_{i}$, not the absolute value of the frequencies. This interesting phenomenon is important for the design of future oscillator-based computing systems. It implies that devices with high frequencies can provide wider bandwidth for information coding. Also, even when the oscillators fail to lock with each other, their frequencies are pulled closer to each other. It is based on this observation that we say that the degree of synchronization can provide a metric to measure the distance or similarity of each oscillator's initial frequency (and thus input) as discussed below.

To verify this point, we run the simulation multiple times by fixing the first oscillator's frequency and sweeping the frequency of the other two from 0.8 to 1.2. We use degree of synchronization, $1-\left\{\left(f_{2}-f_{1}\right)^{2}+\left(f_{3}-f_{1}\right)^{2}\right\}$, as the function to evaluate how well the oscillators synchronize. With this function, we measure how close these final frequencies are to the reference oscillator for comparison between the phase model and direct simulations, and thus to verify the capability of our phase model in simulation of oscillator-based computing. Note that $f_{1}, f_{2}, f_{3}$ here are the coupled frequencies based on normalized phase. In this test, we use the three different methods to obtain the PPV/PRC, as we did in Section 3. In addition, we directly simulate the coupled oscillator with oscillator Equation (16) as a performance standard. Figure 8 demonstrates all the models with 3D plots of the degree of synchronization for the two cases of $\varepsilon=0.4$ and $\varepsilon=0.2$. When the coupling strength is weak, the surface is smooth and the initial frequencies are easier to differentiate, while stronger coupling gives us more nonlinear features and a wider locking range. The flat area on the top of the surface indicates the highest degree of synchronization, giving us the frequency locking range for the simulation sweeps. Hence, a very strong coupling system may lack differentiation for pattern matching or nearest neighbor searching. But for clustering operations like image segmentation, stronger coupling strength can provide better resistance to noise.

Due to the fast simulation speed, our model is also very suitable for simulating systems with large numbers of coupled oscillators. To understand how the number of oscillators can influence the synchronization, we run the simulations with the same two-dimensional frequency sweeping but different numbers of oscillators. Since we cannot show a plot of higher dimensional frequency sweeping, we keep the frequencies of all but two of the oscillators fixed to 1 and sweep the last two. Figure 9 shows the results for $n=3,4,8$, and 16 . In these simulations, we use the analytical PPV as the PPV/PRC function.

From these results we can notice that larger numbers of oscillators with the same frequency gives a larger frequency locking range. Namely, the effective coupling strength to those oscillators with different frequencies becomes stronger because the system's stable state is close to the state of the majority of the oscillators. This could either be an advantage or disadvantage in the design of oscillator-based systems, depending on the application and computation required.

\subsection{Performance and Speedup}

For performance comparisons of our phase model, we calculate the root mean square error between the direct simulation of the oscillators in each case of Figure 9 and the analytical PPV. As we can see in Table I, the error is relatively small compared with the absolute value of degree of synchronization. This shows that our phase model is compatible with different methods for PPV/PRC and robust to the variations between these methods. Compared to the direct oscillator simulation, the analytical PPV 

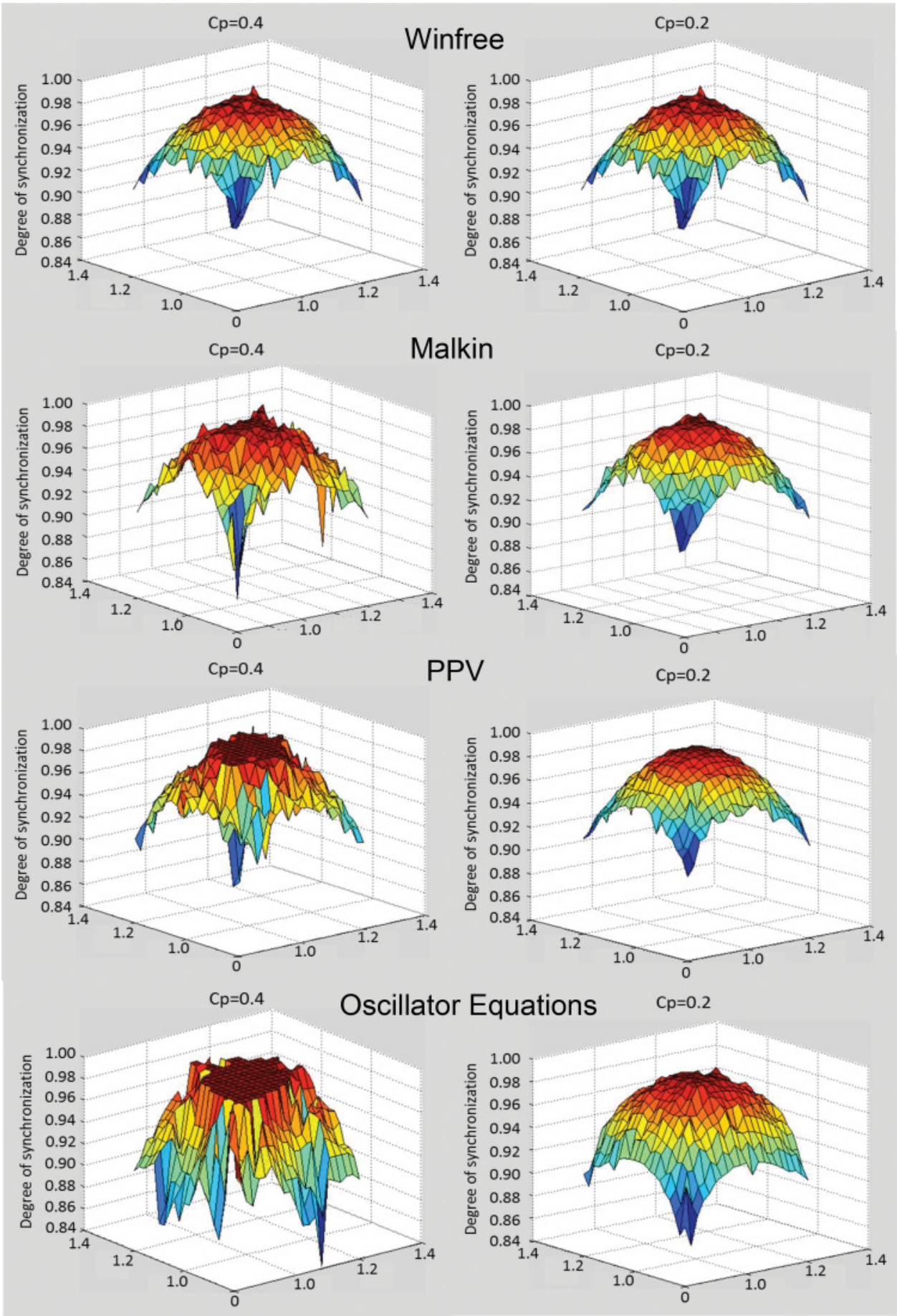

Fig. 8. Degree of synchronization as inverted Euclidean distance, initial frequencies for a range $\lambda_{2,3}=[0.8$ to 1.2] and a fixed frequency of $\lambda_{1}=1.0$, Left column: $\varepsilon=0.4$, Right column: $\varepsilon=0.2$; Row 1 : Winfree's approach, Row 2: Malkin's approach, Row 3: Analytical PPV, Row 4: Direct simulation with oscillator equations. 


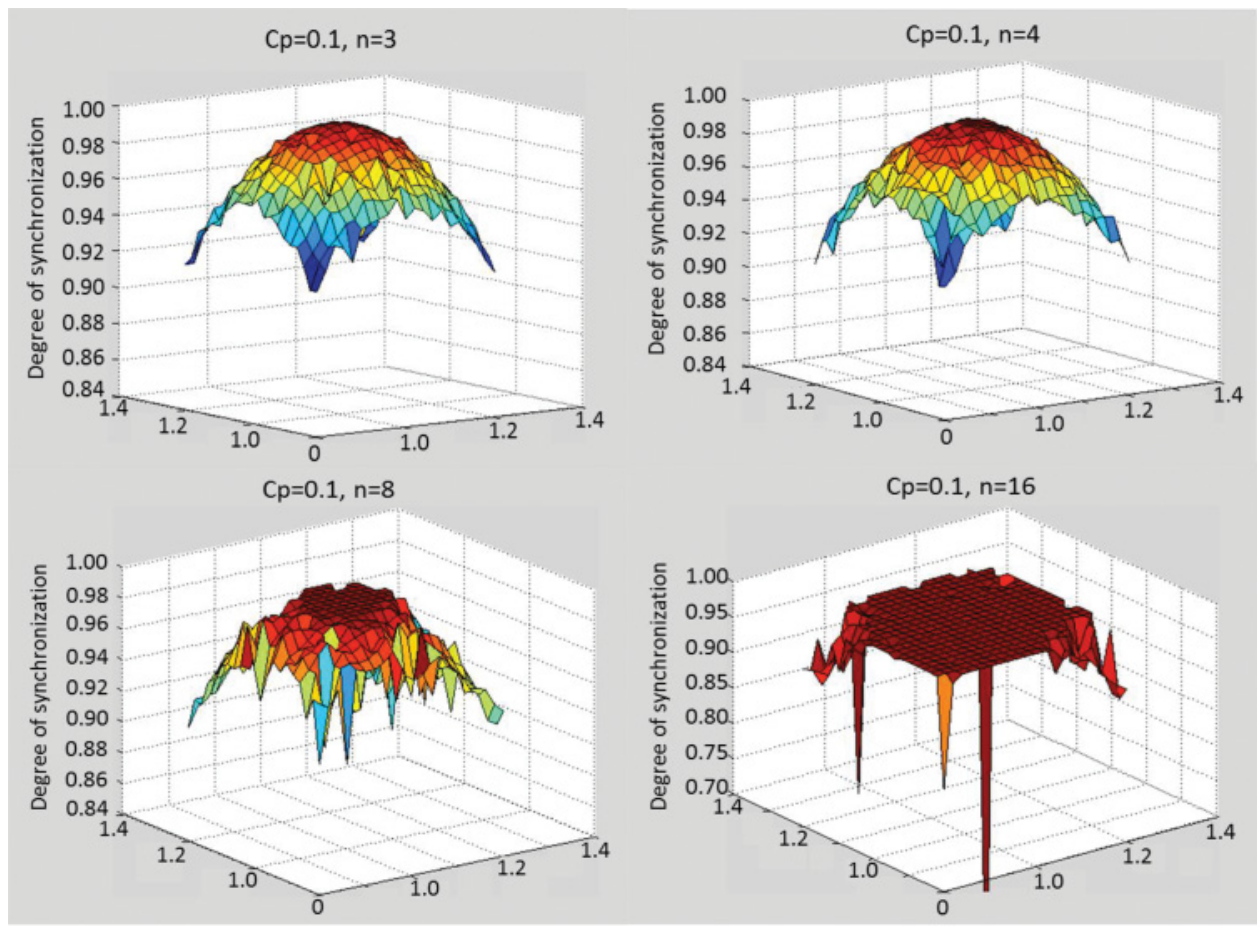

Fig. 9. Simulations of different numbers of coupled oscillators. All oscillators but two are kept at frequency 1 , while the last two are swept.

Table I. RMSE of Simulations Based on Different Methods

\begin{tabular}{|c|c|c|c|}
\hline Comparing & Winfree's & \multicolumn{1}{c|}{ Malkin's } & Analytical PPV \\
\hline Oscillator, $\varepsilon=0.2$ & $127 \mathrm{e}-04$ & $133 \mathrm{e}-04$ & $121 \mathrm{e}-04$ \\
\hline Oscillator, $\varepsilon=0.4$ & $276 \mathrm{e}-04$ & $297 \mathrm{e}-04$ & $271 \mathrm{e}-04$ \\
\hline Analytical PPV, $\varepsilon=0.2$ & $58 \mathrm{e}-04$ & $63 \mathrm{e}-04$ & $/$ \\
\hline Analytical PPV, $\varepsilon=0.4$ & $128 \mathrm{e}-04$ & $142 \mathrm{e}-04$ & $/$ \\
\hline
\end{tabular}

generates the smallest error while Winfree's experimental method gives the largest error, and Malkin's numerical method lies in the middle.

We next evaluate the efficiency of our model by comparing simulation speed to the direct simulation of the oscillator network. The speedup here is defined by the ratio of real time for simulation of two methods (both in Matlab) for the same length of simulation time. Since we always initialize the systems with random phases, we run the test of each configuration 100 times and average the speedup for evaluation. The results in Figure 10 only serve as an approximation of the efficiency of our model because the simulation of coupled oscillator systems is affected by multiple factors, such as the oscillator model, initial states, and the convergence process. Nevertheless, we still observe very promising speedups from our simplified phase model, similar to Lai and Roychowdhury [2005]. The speedup comes from the fact that the differential Equations of the original oscillators are nonlinear but the simplified phase models are linear Equations with much simpler periodic functions. A second advantage is that in the phase domain, simulation is done by fractions of cycles, rather than time steps, so that run time is frequency invariant. This is an advantage in simulation when 


\section{Speedup of Simplfied Phase Model}

\section{0}

1200

1000

800

600

400

200

0
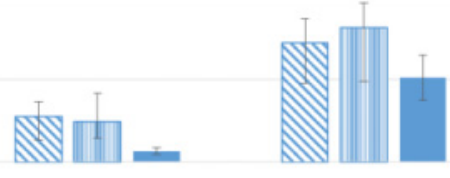

$$
n=3 \quad n=4 \quad n=8 \quad n=16
$$

$\triangle$ Winfree's $\square$ Malkin's Analytical PPV

Fig. 10. Speed-up (factor) of simplified phase model over direct simulation for different PPV/PRC methods.

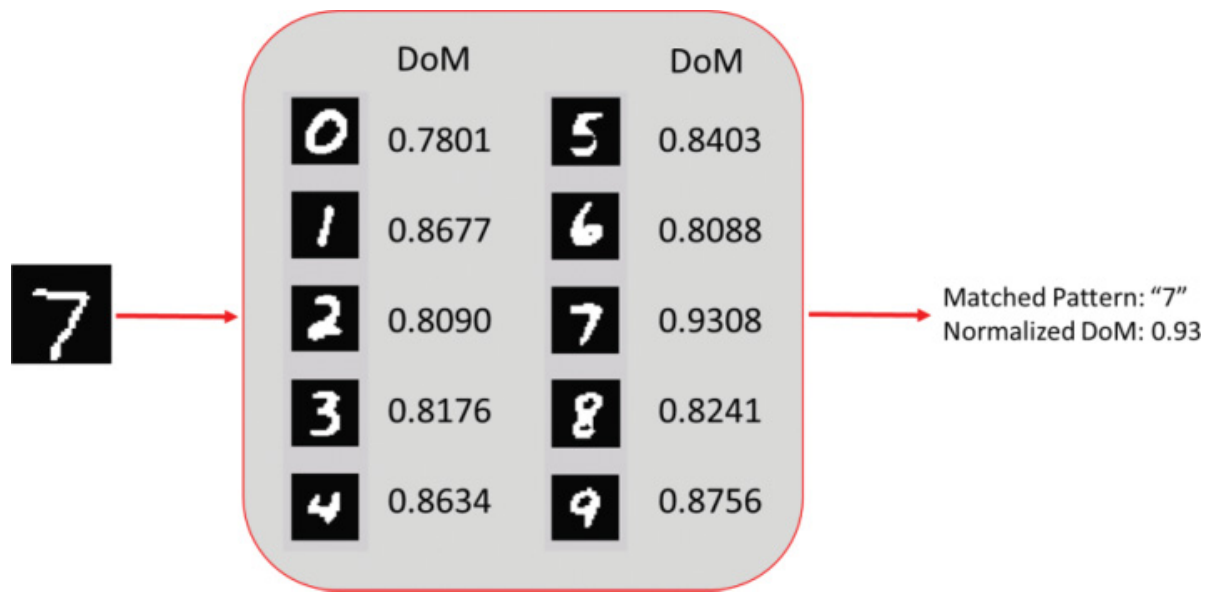

Fig. 11. Pattern matching simulation for handwritten digit recognition and DoM from oscillator clusters.

compared to the regular PPV model where the user must optimize the time step for accurate simulation vs. performance.

\subsection{System Level Simulation}

In order to illustrate the utility of our model for system-level evaluation, we give three examples of the oscillator computing paradigms discussed in Section 2. The first example application is a simple pattern matching task that we discussed in Section 2.1. The goal is to recognize a handwritten digit image from the MNIST dataset, as illustrated in Figure 11. In this task, the stored templates are 10 averaged grayscale images from 10000 classified handwritten images in the training dataset of MNIST [LeCun and Cortes 1998]. The image size is 784 pixels $(28 \times 28)$ and the pixel intensities range from 


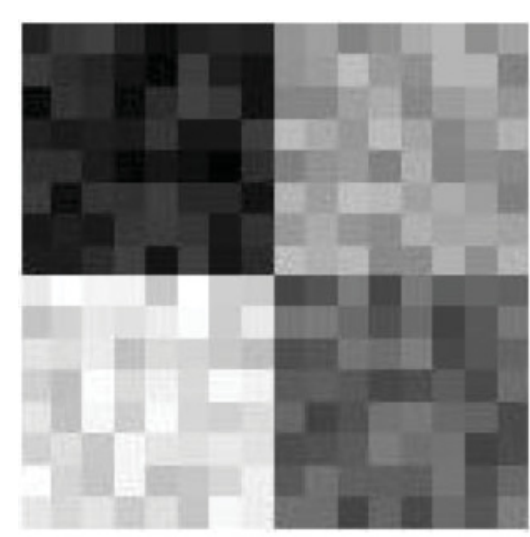

(a)

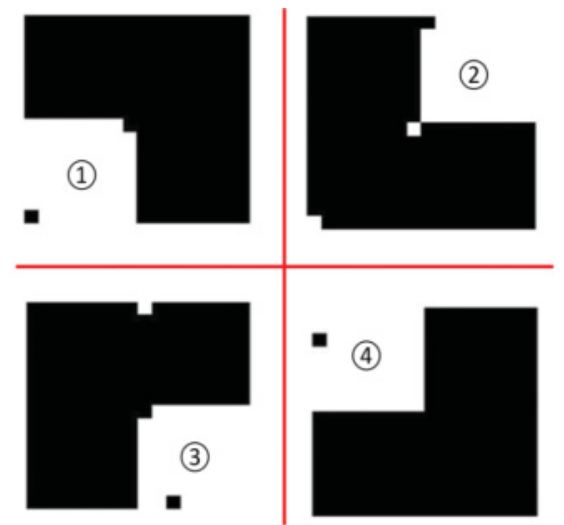

(b)

Fig. 12. (a) Test image with four regions; (b) Segmentation results of each region from oscillator network. Each subsquare is the original image with segmented region labeled as white pixels.

0 to 1 . Each template is the pixel-wise mean of all the images in the corresponding digits binarized with a threshold of 0.4. In our previous work, we directly used the square Euclidean distance rather than the simulation of oscillators [Fang et al. 2015]. The system was evaluated on the MNIST handwritten digit dataset and achieved $94 \%$ accuracy.

For this simulation example, the test input pattern is a "7" randomly picked from the test dataset. We partition the 784 pixels of each image into 49 vectors of 16 pixels each and use our oscillator clusters to perform pattern matching based on these shorter vectors. The reason we partition the image vectors comes from our experiences with oscillator circuits and the challenge we foresee to practical implementations of large scale associative memories. By partitioning each vector, we can overcome the challenges of coupling a large number of oscillators in a symmetric network. For this task, we use a cluster of 17 ring oscillators like the model shown in Figure 2, simulated with the analytic technique for our phase model. One oscillator that is initialized with frequency $=1$ serves as the reference oscillator, meanwhile the remaining oscillators are used to process the vector differences. The normalized oscillator frequencies range from 1 to 1.5 and the coupling coefficient is set to 0.01 . Each oscillator cluster performs a 16-pixel length matching operation. Each comparison between the test image and one template requires 49 such operations, thus the total number of operations for this task is 490 . Figure 11 gives the normalized degrees of match between the input image and the 10 templates with the correct classification. The average simulation speedup for each operation is about 780 compared to simulation with the original oscillator equations.

The second system simulation example is the image segmentation mentioned in Section 2.2. In this test, we generate a $16 \times 16$ grayscale image that is composed of 4 regions with different intensity ranges, as shown in Figure 12(a). The objective of this test is to separate each region from the others. Each region is composed of 64 randomly placed pixels with intensities from one quarter of the $0 \sim 1$ range. All 256 pixels have unique intensities and thus the intensity histogram is a uniform distribution, which is difficult for those segmentation algorithms that are solely based on intensity histograms. We apply the oscillator structure given in Figure 3(a) to this task, setting the oscillator coupling strength to 0.2 and initializing the oscillator frequencies in the range of $1 \sim 2$ with associated pixel intensities. When the system evolves to a stable state, the oscillators cluster into four groups by frequency, corresponding to four image regions. The central frequencies of the oscillators in these four regions shown in Figure 12(b) are 

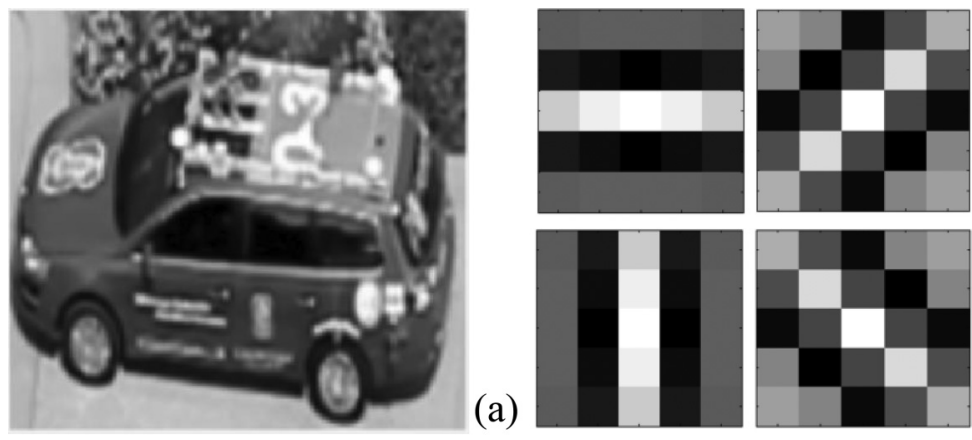

(a)

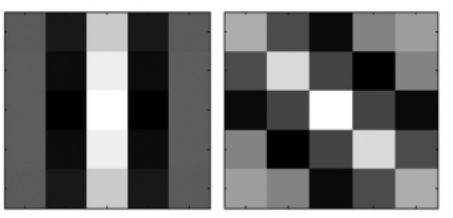

(b)
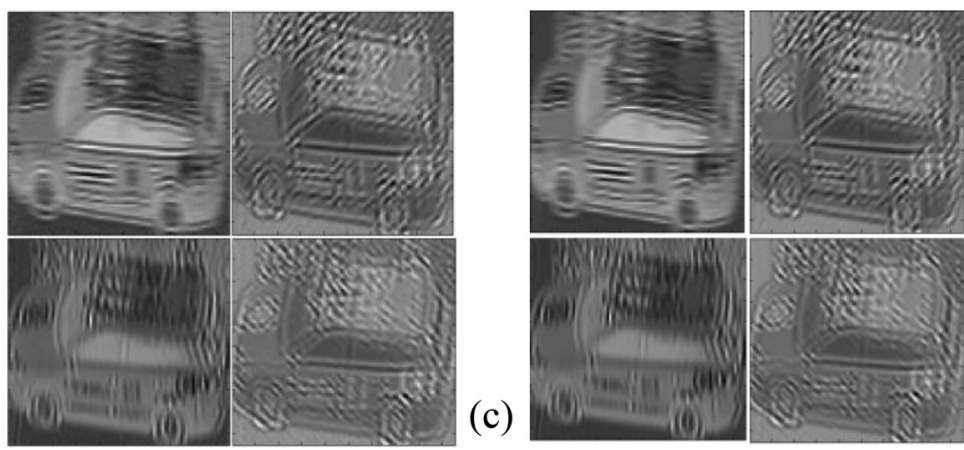

(d)

Fig. 13. Using $5 \times 5$ Gabor filter to filter the "Car" image. (a) Test image; (b) Gabor filter bank; (c) Results from Oscillator Cluster; (d) Results from digital 2-D convolution.

[1.121 1.367 1.634 1.891] and the frequency band of each group is around 0.2. Thus, we can obtain each segmented region by band-pass filtering the output signals of the oscillators in an associated frequency range. Figure 12(b) shows the segmentation results of the four regions with corresponding region numbers. This result indicates that the oscillator network is clustering the pixels based on both intensity and locality. In this simulation, the estimated speedup is about 300 for each oscillator and its neighbors compared to using the direct simulation using oscillator equations.

Our third example is a convolution operation for image filtering [Jennings et al. 2014]. Figure 13 illustrates the process of our simulation. The test image is a $100 \times$ 100 pixel grayscale "car" image Figure 13(a). To test our oscillator-based convolution model, we perform a 2-D convolution between the test image and a $5 \times 5$ Gabor filter bank with four directions (0, 45, 90, and 135 degrees). Figure 13(b) displays grayscale images of these filters. Gabor filters are commonly used for edge detection and feature extraction in image processing. As we discussed in Section 2.2, the convolution of two vectors can be implemented by three oscillator clusters. In this case, each pixel in the output image is computed by the pair-wise multiplication between an image patch and a filter mask. We use three 26-oscillator clusters (each cluster has one oscillator for reference) to compute the values of $(\mathrm{A}-\mathrm{B})^{2}, \mathrm{~A}^{2}$, and $\mathrm{B}^{2}$. The frequency range is $1 \sim 1.5$ with a relatively small coupling coefficient of 0.002 . The filtered images from the oscillator system and a 2-D convolution function in Matlab are respectively depicted in Figures 13(c) and 13(d). We notice that the simulation result of oscillator-based convolution is very similar to the result of the Matlab function. In this operation, the approximate speedup of each operation is about 1120 compared to the direct simulation with oscillator equations. 
These three examples of system-level simulation for oscillator-based computing have demonstrated that our phase model can greatly improve the simulation efficiency for large oscillator networks, while maintaining acceptable accuracy for the evaluation of these applications.

\section{SUMMARY AND CONCLUSIONS}

In this article, we show the promise of oscillator-based computing by giving three examples of different applications in image processing and computer vision. We describe the problems of coupled oscillator simulations for system design. To address this problem, we propose a simplified phase model and apply this model to the analysis of coupled ring oscillators. The results indicate that our model is capable of predicting the frequency and phase of coupled oscillator systems with small errors compared to the direct simulation of the oscillator model. We demonstrate that this model is particularly suitable for simulation and analysis for oscillator based applications such as pattern matching, segmentation, and convolution. The main contribution of our model is in simplifying the nonlinear equations and transferring simulation from the traditional time domain into the phase domain. This provides several orders of magnitude simulation speedup for large size systems. For these three application examples, the speedup factors obtained from the simplified model are respectively 780, 300, 1120.

\section{REFERENCES}

G. I. Bourianoff, J. E. Brewer, R. Cavin, J. A. Hutchby, and V. Zhirnov. 2008. Boolean logic and alternative information-processing devices. Computer 41 5, 38-46.

D. M. Chiarulli, B. B. Jennings, Y. Fang, A. Seel, and S. P. Levitan. 2015. A computational primitive for convolution based on coupled oscillator arrays. In Proceedings of the 2015 IEEE Computer Society Annual Symposium on VLSI (ISVLSI).

M. J. Cotter, Y. Fang, S. P. Levitan, D. M. Chiarulli, and V. Narayanan. 2014. Computational architectures based on coupled oscillators. In Proceedings of the 2014 IEEE Computer Society Annual Symposium on VLSI (ISVLSI), 130-135.

G. Csaba and W. Porod. 2013. Computational study of spin-torque oscillator interactions for non-Boolean computing applications. IEEE Transactions on Magnetics 49, 7, 4447-4451.

A. Demir, A. Mehrotra, and J. Roychowdhury. 2000. Phase noise in oscillators: a unifying theory and numerical methods for characterization. IEEE Transactions on Circuits and Systems I: Fundamental Theory and Applications, 47, 5, 655-674.

D. Fan, S. Maji, K. Yogendra, M. Sharad, and K. Roy. 2015. Injection-locked spin hall-induced coupledoscillators for energy efficient associative computing. IEEE Transactions on Nanotechnology 14, 6, 10831093.

Y. Fang, M. J. Cotter, D. M. Chiarulli, and S. P. Levitan. 2014. Image segmentation using frequency locking of coupled oscillators. In Proceedings of the 2014 14th International Workshop on Cellular Nanoscale Networks and Their Applications (CNNA), IEEE, 1-2.

Y. Fang, S. P. Levitan, D. M. Chiarulli, and D. H. Dash. 2015. Alternative architectures for non-Boolean information processing systems. In Emerging Nanoelectronic Devices. John Wiley and Sons Ltd., 467497.

C. C. Foster, 1976. Content addressable parallel processors, John Wiley and Sons, Inc., New York.

F. C. Hoppensteadt and E. M. Izhikevich. 1997. Weakly Connected Neural Networks. Springer-Verlag, New York.

F. C. Hoppensteadt and E. M. Izhikevich. 2000. Pattern recognition via synchronization in phase-locked loop neural networks. IEEE Transactions on Neural Networks, 3, 734-738.

J. A. Hutchby, R. Cavin, V. Zhirnov, J. E. Brewer, and G. I. Bourianoff. 2008. Emerging nanoscale memory and logic devices: A critical assessment. Computer 41, 5, 28-32.

B. B. Jennings, R. Barnett, C. Gnegy, J. Carpenter, Y. Fang, D. M. Chiarulli, and S. P. Levitan. 2014. HMAX image processing pipeline with coupled oscillator acceleration. In Proceedings of the 2014 IEEE Workshop on Signal Processing Systems (SiPS) 1-6.

M. Kabir and M. Stan. 2014. Computing with hybrid CMOS/STO circuits. In Proceedings of the 51st Annual Design Automation Conference on Design Automation Conference, ACM, 1-6. 
A. Krizhevsky, I. Sutskever, and G. E. Hinton. 2012. Imagenet classification with deep convolutional neural networks. In Advances in Neural Information Processing Systems. 1097-1105.

Y. Kuramoto 1984. Chemical Oscillations, Waves, and Turbulence. Springer.

X. Lai and J. Roychowdhury. 2005. Analytical equations for predicting injection locking in LC and ring oscillators. In Proceedings of the IEEE Custom Integrated Circuits Conference, 2005, 461-464.

Y. LeCun and C. Cortes. 1998. MNIST handwritten digit database. AT\&T Labs [Online]. Available: http://yann.lecun.com/exdb/mnist/.

S. P. Levitan, Y. Fang, D. H. Dash, T. Shibata, D. E. Nikonov, and G. I. Bourianoff. 2012. Non-Boolean associative architectures based on nano-oscillators. In Proceedings of the 13th IEEE International Workshop on Cellular Nanoscale Networks and Their Applications (CNNA), (1-6).

N. Locatelli, V. Cros, and J. Grollier. 2014. Spin-torque building blocks. Nature Materials 13, 1, 11-20.

Neovision2 dataset - iLab - University of Southern California. Retrieved November 12, 2013, from http://ilab. usc.edu/neo2/dataset/, 2013.

Paolo. Maffezzoni. 2010. Synchronization analysis of two weakly coupled oscillators through a PPV macromodel. IEEE Transactions on Circuits and Systems I: Regular Papers 57.3, 654-663.

M. R. Pufall, W. H. Rippard, S. Kaka, T. J. Silva, and S. E. Russek. 2005. Frequency modulation of spintransfer oscillators, Applied Physics Letters 86, 8, 082506-082506.

T. Serre and M. Riesenhuber. 2004. Realistic modeling of simple and complex cell tuning in the HMAX model, and implications for invariant object recognition in cortex, CBCL Paper 239/AI Memo 2004-017, Massachusetts Institute of Technology, Cambridge, 2004.

T. Shibata, R. Zhang, S. P. Levitan, D. E. Nikonov, and G. I. Bourianoff, 2012. CMOS supporting circuitries for nano-oscillator-based associative memories. In Proceedings of the 13th IEEE International Workshop on Cellular Nanoscale Networks and Their Applications (CNNA), 1-5.

N. Shukla, A. Parihar, E. Freeman, H. Paik, G. Stone, V. Narayanan, H. Wen, Z. Cai, V. Gopalan, R. EngelHerbert, D. G. Schlom, A. Raychowdhury, and S. Datta. 2014. Synchronized charge oscillations in correlated electron systems. Scientific Reports 4.

E. M. Izhikevich. 2007. Synchronization, Chapter 10 In Dynamical Systems in Neuroscience. MIT press.

D. Wang and D. Termani. 1995. Locally excitatory globally inhibitory oscillator networks. IEEE Transactions on Neural, 1, 283-286.

D. Weinstein and S. A. Bhave. 2010. The resonant body transistor. Nano Letters, 4, 1234-1237.

A. T. Winfree. 1967. Biological rhythms and the behavior of populations of coupled oscillators. J. Theoret., $16,1,15-42$.

Received September 2015; revised February 2016; accepted July 2016 